\title{
Photonic Generation of Ultra-Wideband Signals via Pulse Compression in a Highly Nonlinear Fiber
}

\author{
Avi Zadok, Xiaoxia Wu, Student Member, IEEE, Jacob Sendowski, Amnon Yariv, Life Fellow, IEEE, and \\ Alan E. Willner, Fellow, IEEE
}

\begin{abstract}
A simple and reconfigurable scheme for photonic generation of ultra-wideband (UWB) waveforms is proposed, simulated, and demonstrated. The technique relies on pulse compression in a highly nonlinear fiber and subsequent differential detection. A center frequency of $25 \mathrm{GHz}$ and a fractional bandwidth of $120 \%$ are obtained. The generated waveform is also adjusted to closely approximate the spectral mask for unlicensed UWB indoor wireless communications.
\end{abstract}

Index Terms-Microwave photonics, nonlinear fiber-optics, ultra-wideband (UWB) pulse generation.

\section{INTRODUCTION}

$\mathbf{U}$ LTRA-WIDEBAND (UWB) technology has attracted much attention recently due to its low power consumption, immunity to multipath fading, potential for interference mitigation, and compatibility with traditional, narrowband communication systems [1]. Potential applications include wireless communications, sensor networks, location tracking systems, and radars [1]. Microwave photonics-based techniques for generating UWB pulses have the advantages of large processing bandwidth, reconfigurable waveform shape, immunity to electromagnetic interference, and parallel processing via wavelength multiplexing. Indoor UWB wireless communication systems, in particular, represent an attractive application for photonic-based techniques. The wireless transmission reach in such systems is only a few meters, and it might be extended through fiber-based distribution and broadcasting as part of a network solution [2].

Manuscript received June 29, 2009; revised October 06, 2009; accepted November 25, 2009. First published January 12, 2010; current version published January 27, 2010. This work was supported in part by the National Science Foundation (NSF) ERC CIAN. The work of A. Zadok was supported by a postdoctoral fellowship from the Center for the Physics of Information (CPI), California Institute of Technology.

A. Zadok was with the California Institute of Technology, Pasadena, CA 91125 USA. He is now with the School of Engineering, Bar-Ilan University, Ramat-Gan 52900, Israel (e-mail: zadoka@eng.biu.ac.il).

X. Wu and A. E. Willner are with the Department of Electrical EngineeringSystems, University of Southern California, Los Angeles, CA 90089-2565 USA (e-mail: xiaoxia@usc.edu; willner@usc.edu).

J. Sendowski is with the Department of Electrical Engineering, California Institute of Technology, Pasadena, CA 91125 USA (e-mail: jacobsen@ caltech. edu).

A. Yariv is with the Department of Electrical Engineering and the Department of Applied Physics, California Institute of Technology, Pasadena, CA 91125 USA (e-mail: ayariv@caltech.edu)

Color versions of one or more of the figures in this letter are available online at http://ieeexplore.ieee.org.

Digital Object Identifier 10.1109/LPT.2009.2037929
Numerous avenues have been followed towards optical UWB pulse generation in recent years. Wang et al. used cross-gain modulation in semiconductor optical amplifiers to generate monocycle Gaussian pulses [3]. The technique was successfully extended to the generation of doublet pulses with the use of polarization modulation and a differential group delay element, though the scheme required a special phase modulator and polarization control [4]. Moreover, elaborate tailoring of the pulse waveform was demonstrated using: 1) multitap microwave photonic filters [5], and 2) spectral shaping and subsequent frequency-to-time mapping [6]-[8]. Implementations of these methods required either multiple tunable laser sources and modulators [5], or mode-locked fiber lasers [6]-[8] and highly specialized spectral components [7], [8].

The UWB pulse generation technique described in this work relies on self-propagation nonlinearity in an optical fiber, and therefore, requires only a single laser source and a single optical modulator. Gaussian pulses are spectrally broadened by selfphase modulation (SPM) in a commercially available highly nonlinear fiber (HNLF), and then temporally narrowed through optical bandpass filtering. This powerful pulse shaping technique, originally proposed for optical regeneration of data [9], is applied to UWB generation in this work. The compressed pulses are subtracted from replicas of the original ones using a differential detector. The obtained waveform is conveniently reconfigurable through several methods: adjusting the input power, tuning the optical filter, or controlling the delay and attenuation of the reference pulses. A recent demonstration of UWB pulses generation in an HNLF was based on a parametric amplification process, and accordingly required two laser sources [10].

The proposed technique is demonstrated both via simulations and experiments. Doublet-shaped UWB waveforms having a central frequency of $25 \mathrm{GHz}$ and a fractional bandwidth of $120 \%$ are experimentally generated. Using a different set of parameters, the obtained pulses come close to accommodating the requirements of the U.S. Federal Communications Commission (FCC) approved spectral mask for unlicensed, indoor UWB wireless communications [11].

\section{PRINCIPLE OF OPERATION}

A diagram of the proposed UWB pulse generation scheme is shown in Fig. 1. Light from a tunable laser source at an optical frequency of $\omega_{0}$ is modulated by a train of Gaussian pulses, each having a full-width at half-maximum (FWHM) of $T_{0}$, and then split into two paths. The pulses in the upper branch are amplified by a high-power erbium-doped fiber amplifier (EDFA) to a peak power of $P_{0}$, and launched into an HNLF of length 


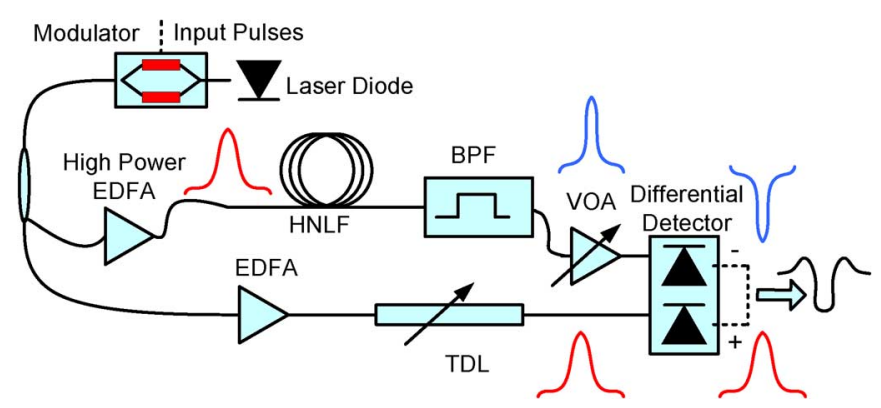

Fig. 1. Schematic diagram of the UWB doublet pulse generation. EDFA: Erbium-doped fiber amplifier.
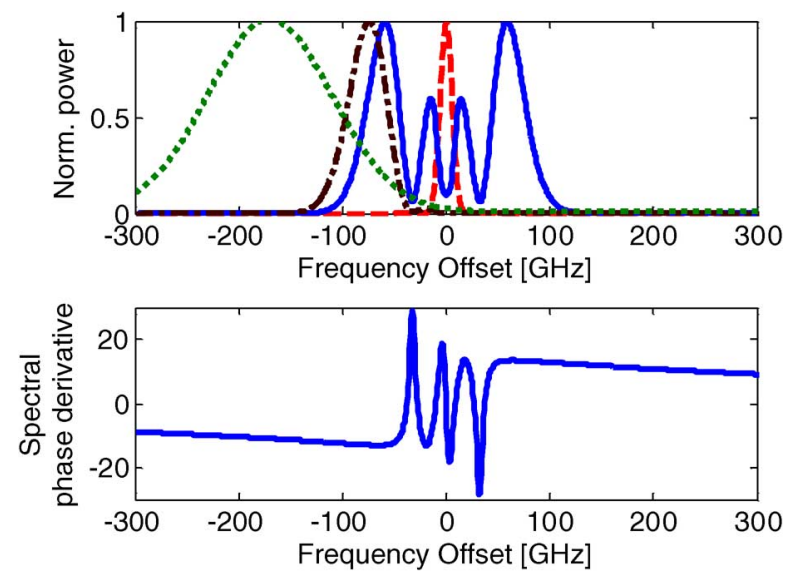

Fig. 2. (Top) Calculated, normalized optical power spectra at the HNLF input (dashed), HNLF output (solid), and BPF output (dashed-dotted). The dotted curve denotes the BPF magnitude response. (Bottom) Calculated derivative of the spectral phase $d \varphi(\omega) / d \omega$ [ps] at the HNLF output. Simulation parameters were: $L=1 \mathrm{~km}, \gamma=11.3[\mathrm{~W} \cdot \mathrm{km}]^{-1}, P_{0}=1 \mathrm{~W}, T_{0}=33 \mathrm{ps}$, $\Delta \omega_{\mathrm{BPF}} / 2 \pi=140 \mathrm{GHz}, \delta \omega_{\mathrm{BPF}} / 2 \pi=170 \mathrm{GHz}$.

$L$ and nonlinear coefficient $\gamma[\mathrm{W} \cdot \mathrm{km}]^{-1}$. We denote the optical field as a function of frequency $\omega$ at the HNLF output as: $E_{\mathrm{HNLF}}(\omega)=A(\omega) \exp [j \varphi(\omega)]$, where the real-valued $A(\omega)$ and $\varphi(\omega)$ represent the spectral amplitude and phase, respectively. The optical power spectrum $|A(\omega)|^{2}$ is broadened by SPM (see the top of Fig. 2).

The waveform at the HNLF output passes through an optical bandpass filter (BPF) of bandwidth $\Delta \omega_{\mathrm{BPF}}>1 / T_{0}$. The BPF can be detuned by $\delta \omega_{\mathrm{BPF}}$ from $\omega_{0}$ to select an outer spectral side-lobe, which is broader than the original spectral width, and suppress the central lobes (see Fig. 2). The filtered waveform, in this case, becomes a train of compressed pulses. Theoretically, the output pulsewidth is inversely proportional to the filter bandwidth [9]. Note that the spectral phase derivative $d \varphi(\omega) / d \omega$ is nearly constant within the spectral sidelobes of the pulse (bottom of Fig. 2), so that the compressed pulses are practically chirp-free. The optical power of the compressed pulses is subtracted from that of a reference pulse train, propagating in the lower branch, using a balanced differential photodetector. The relative group delay and the ratio of peak power levels between the pulses in the two branches are balanced by a tunable optical delay line (TDL) and a variable optical attenuator (VOA), respectively.

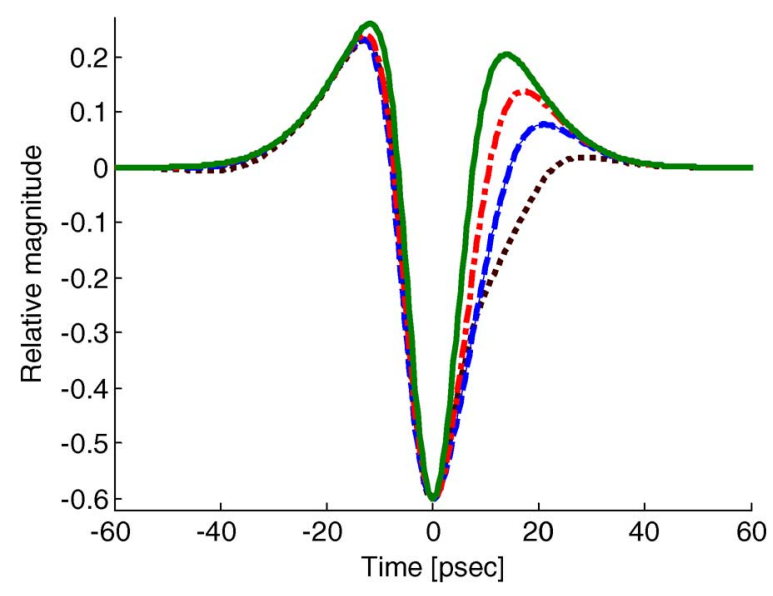

Fig. 3. Examples of calculated output pulse shapes $\Delta P(t)$. For all curves: $L=1 \mathrm{~km}, \gamma=11.3[\mathrm{~W} \cdot \mathrm{km}]^{-1}, P_{0}=1 \mathrm{~W}, T_{0}=33 \mathrm{ps}, \Delta \omega_{\mathrm{BPF}} / 2 \pi=$ $140 \mathrm{GHz}$. The BPF detuning $\delta \omega_{\mathrm{BPF}} / 2 \pi$ was $60 \mathrm{GHz}$ (black, dotted), $90 \mathrm{GHz}$ (blue, dashed), $120 \mathrm{GHz}$ (red, dashed-dotted), and $170 \mathrm{GHz}$ (green, solid).
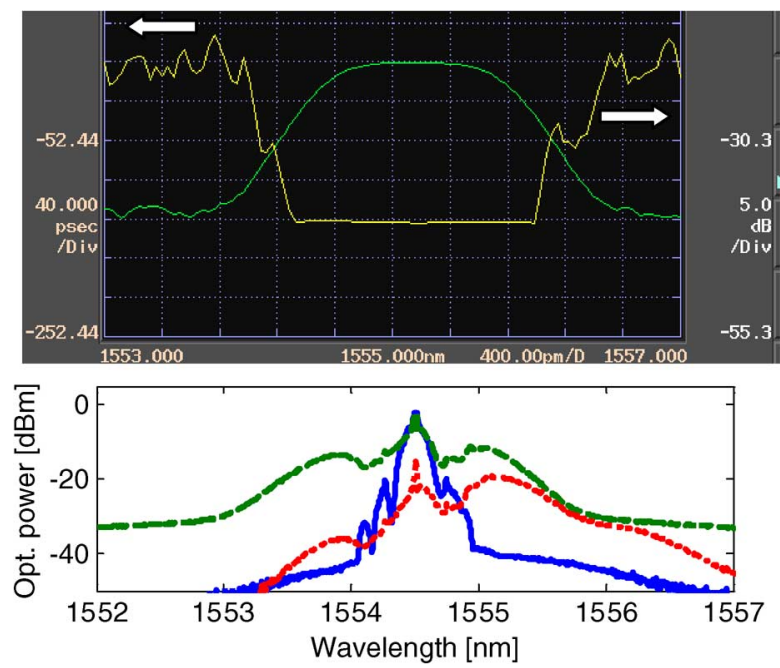

Fig. 4. (Top) Measured magnitude response and group delay of the BPF as a function of wavelength. (Bottom) Optical spectrum analyzer measurements of the pulse train at the HNLF input (blue, solid), HNLF output (green, dahsed), and BPF output (red, dotted). $P_{0}=1 \mathrm{~W}, L=1 \mathrm{~km}, \gamma=11.3[\mathrm{~W} \cdot \mathrm{km}]^{-1}$, $\Delta \omega_{\mathrm{BPF}} / 2 \pi=140 \mathrm{GHz}, \delta \omega_{\mathrm{BPF}} / 2 \pi=170 \mathrm{GHz}, T_{0}=33 \mathrm{ps}$.

The detected power-difference waveform $\Delta P(t)$ can be modified through changing $T_{0}, P_{0}$, and $\Delta \omega_{\mathrm{BPF}}$, as well as via control of the VOA and TDL. Since the BPF magnitude response is chosen to be nonuniform within the selected spectral sidelobe, $\Delta P(t)$ can also be modified by adjusting the detuning $\delta \omega_{\mathrm{BPF}}$, as illustrated in Fig. 3. With a proper choice of parameters $\Delta P(t)$ can provide various UWB pulse shapes. In particular, $\Delta P(t)$ can be adjusted to approximate a doublet shape, as demonstrated in Section III.

\section{EXPERIMENT}

The generation of UWB pulses was demonstrated experimentally. The pulsewidth and peak power at the HNLF input, the HNLF length and nonlinear coefficient and the BPF bandwidth were the same as those of the simulations of Fig. 3, with the filter detuning $\delta \omega_{\mathrm{BPF}} / 2 \pi$ set to $170 \mathrm{GHz}$. The magnitude response and group delay of the BPF are shown in Fig. 4 (top). The rate 

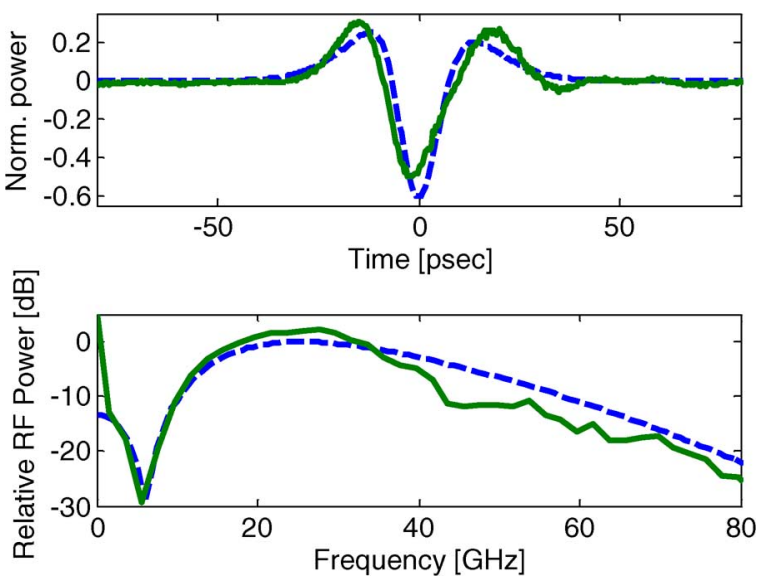

Fig. 5. (Top) Measured (solid) and simulated (dashed) $\Delta P(t)$. (Bottom) Calculated RF spectrum of measured $\Delta P(t)$ (solid), and the corresponding simulated spectrum (dashed). For both panels: $P_{0}=1 \mathrm{~W}, L=1 \mathrm{~km}, \gamma=$ $11.3[\mathrm{~W} \cdot \mathrm{km}]^{-1}, \Delta \omega_{\mathrm{BPF}} / 2 \pi=140 \mathrm{GHz}, \delta \omega_{\mathrm{BPF}} / 2 \pi=170 \mathrm{GHz}, T_{0}=$ 33 ps.

of the pulses was $3.75 \mathrm{GHz}$. The path lengths of the HNLF and reference arm were not matched. An operational system would require path length equalization to an accuracy of several millimeters, which may necessitate shorter fiber spans with higher peak power levels or thermal isolation. Fig. 4 (bottom) presents the measured optical power spectra at the HNLF input, HNLF output, and BPF output.

Fig. 5 shows the measured $\Delta P(t)$ (top) and its calculated radio-frequency (RF) power spectrum (bottom), alongside the corresponding simulation results. The center frequency of the output waveform was $25 \mathrm{GHz}$, and the fractional bandwidth, defined as the ratio of the $-10 \mathrm{~dB}$ full spectral width to the central frequency, was $120 \%$. The small difference between simulation and experiment may be attributed to distortion induced by the nonlinear interaction between the signal and amplified spontaneous emission noise [12], and to the deviations of the input pulse from the ideal Gaussian shape.

With the use of a narrower BPF, wider input pulses, and a higher peak power at the HNLF input, the output waveform can come close to accommodating the FCC spectral mask requirements (Fig. 6). The pulses rate in this experiment was $1.25 \mathrm{GHz}$. The mask infringement at the lower frequency range may be corrected by choosing a slightly different value of $\Delta \omega_{\mathrm{BPF}}$, unavailable to us in the preliminary experiment.

\section{CONCLUSION}

A simple technique for the photonic generation of UWB pulses has been proposed and demonstrated. The technique is based on pulse compression in a nonlinear optical fiber, which may also serve for the pulses distribution, and does not require specialty components. The underlying mechanism used is SPM, so that only a single laser source is required. The pulse shape obtained is conveniently reconfigurable. The technique is scalable to the generation of more complicated pulse shapes,
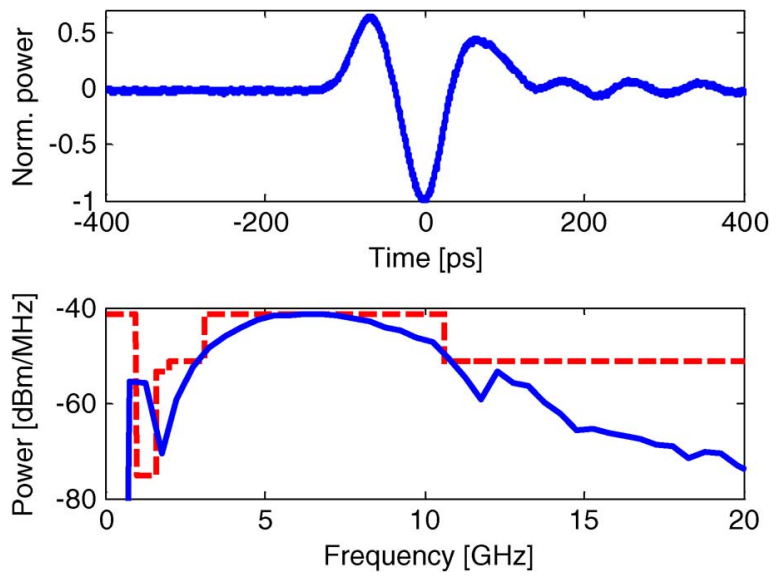

Fig. 6. (Top) Measured $\Delta P(t)$. (Bottom) Calculated RF power spectrum of measured $\Delta P(t)$ (solid), alongside the FCC mask for indoor UWB communication [9] (dashed). For both panels: $P_{0}=2 \mathrm{~W}, L=1 \mathrm{~km}, \gamma=11.3[\mathrm{~W} \cdot \mathrm{km}]^{-1}$, $\Delta \omega_{\mathrm{BPF}} / 2 \pi=40 \mathrm{GHz}, \delta \omega_{\mathrm{BPF}} / 2 \pi=30 \mathrm{GHz}, T_{0}=100 \mathrm{ps}$.

through using multiple BPFs to carve several compressed replicas of the input pulses.

\section{REFERENCES}

[1] L. Yang and G. B. Giannakis, "Ultra-wideband communications: An idea whose time has come," IEEE Signal Process. Mag., vol. 21, no. 6, pp. 26-54, Nov. 2004.

[2] J. Yao, F. Zeng, and Q. Wang, "Photonic generation of ultrawideband signals," J. Lightw. Technol., vol. 25, no. 11, pp. 3219-3235, Nov. 2007.

[3] Q. Wang, F. Zeng, S. Blais, and J. Yao, "Optical ultrawideband monocycle pulse generation based on cross-gain modulation in a semiconductor optical amplifier," Opt. Lett., vol. 31, no. 21, pp. 3083-3085, Nov. 2006.

[4] H. Chen, M. Chen, T. Wang, M. Li, and S. Xie, "Methods for ultrawideband pulse generation based on optical cross-polarization modulation," J. Lightw. Technol., vol. 26, no. 15, pp. 2492-2499, Aug. 1, 2008.

[5] M. Bolea, J. Mora, B. Ortega, and J. Capmany, "Optical UWB pulse generator using an $\mathrm{N}$ tap microwave photonic filter and phase inversion adaptable to different pulse modulation formats," Opt. Express, vol. 17, no. 7, pp. 5021-5032, Mar. 2009.

[6] C. Wang, F. Zeng, and J. Yao, "All-fiber ultrawideband pulse generation based on spectral shaping and dispersion-induced frequency-to-time conversion," IEEE Photon. Technol. Lett., vol. 19, no. 3, pp. 137-139, Feb. 1, 2007.

[7] J. D. McKinney, I. S. Lin, and A. M. Weiner, "Shaping the power spectrum of ultra-wideband radio-frequency signals," IEEE Trans. Microw. Theory Tech., vol. 54, no. 12, pt. 1, pp. 4247-4255, Dec. 2006.

[8] M. Abtahi, M. Mirshafiei, J. Magne, L. A. Rusch, and S. LaRochelle, "Ultra-wideband waveform generator based on optical pulse-shaping and FBG tuning," IEEE Photon. Technol. Lett., vol. 20, no. 2, pp. 135-137, Jan. 15, 2008.

[9] P. V. Mamyshev, "All optical data regeneration based on self-phase modulation effect," in Proc. Eur. Conf. Optical Communication (ECOC '98), Madrid, Spain, Sep. 1998, pp. 475-476.

[10] J. Li, B. P.-P. Kuo, and K. K.-Y. Wong, "Ultra-wideband pulse generation based on cross-gain modulation in fiber optical parametric amplifier,' IEEE Photon. Technol. Lett., vol. 21, no. 4, pp. 212-214, Feb. 15, 2009.

[11] D. Porcine, P. Research, and W. Hirt, "Ultra-wideband radio technology: Potential and challenges ahead," IEEE Commun. Mag., vol. 41, no. 7, pp. 66-74, Jul. 2003.

[12] H. Kubota, K. R. Tamura, and M. Nakazawa, "Analysis of coherence maintained ultrashort optical pulse trains and supercontinuum generation in the presence of soliton-amplified spontaneous emission interaction," J. Opt. Soc. Amer. B, vol. 16, no. 12, pp. 2223-2232, Dec. 1999. 